\title{
KLEINERE ALTENGLISCHE DICHTUNGEN.
}

An verschiedenen orten finden sich ae. verse und kleinere gedichte zerstreut, die in Wülkers neuausgabe von Greins Bibliothek der ags. Poesie fehlen und deren sammlung angebracht sein dürfte. Was mir von solchen stücken bekannt geworden ist, gebe ich hier in kritisch hergestelltem text. ${ }^{1}$ )

I. Sprüche.

Im 1. bande der Anglia s. 285 f. veröffentlichte Zupitza aus der hs. Cott. Faust. A X des brit. museums folgende "lateinisch - englische sprüche":

1. hāt ācōlad, hwīt assōlad, leof ālāðab, leoht ājōystrað.

Ardor frigescit, nitor squalescit, amor abolescit, lux obtenebrescit.

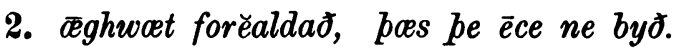

Senescunt omnia, quae aeterna non sunt.

Zup. weist darauf hin, dals die ersten beiden halbverse dem v. 67 des Reimliedes ${ }^{2}$ ) entsprechen:

sěaro-hwīt sōlad, sumur-hāt cōlad.

Die beiden ersten verse hat dann Wülker ib. II, $373 \mathrm{f}$. auch aus der Londoner hs. Roy. Ms. 2 B. V mitgeteilt:

hāt ācōlad, hwit āsōlað, leof āläbad, leoht āpeostrađ (-ad hs).

1) Die beiden gedichte in Alfreds übersetzung der Cura past. sind in Zupitza - Schippers übungsbuche bequem zugänglich.

2) Von mir neu herausgegeben in der Festschr. für Morsbach, Halle 1913 , s. $190 \mathrm{ff}$. 
HOLTHAUSEN, KLEINERE ALTENGLISCHE DICHTUNGEN. 401

Der latein. text entspricht auch genau, nur dals diese hs. refriescit bietet.

Desgleichen der zweite spruch mit einer kleinen abweichung:

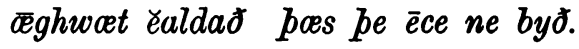

\section{Gloria.}

Drei verszeilen aus der hs. Cott. Tit. D 27 des 11. jahrh. teilt Sievers in der Zeitschr. f. d. Alt. 21, 190 mit:

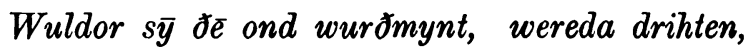
foeder, on foldan, foegere gemëene mid sylfan sunu ond sōðum gāste! amen.

III. Inschrift des Runenkästchens.

$\mathrm{Zu}$ den schon früher bekannten zwei versen auf dem runenkästchen des brit. museums sind durch die publikationen von Wadstein ${ }^{1}$ ), Napier ${ }^{2}$ ) und Vietor ${ }^{3}$ ) noch drei hinzugekommen, die so lauten:

Hèr ho[r]s sitoep on harmberga, $\bar{a} g l[\bar{a} c]$ drīgip, swē hiri ertae gisgrāf säer den sorgce and sefa tornace.

sitæep steht für sitip, hørm für hěarm oder harm, ertae für Erca, gisgräf für giscrā $f$, den für end 'und', sefa für sefan, tornce ist $=$ tornnce (ak. sg. m.); hiri steht für hirce und kann nur reflexiver dativus ethicus sein, wenn man nicht verschreibung für him (auf hors bezüglich) annehmen will; ertae ist $=$ Ercoe in dem bekannten zauberspruch, der mit Erce, Erce, ěorðan modor beginnt (Stevenson bei Napier p. 375 anm. 4); die form end für and begegnet in einer prosainschrift des kåstchens: Titus end Giupeasu (!) und ist zuerst von Imelmann so gedeutet worden; sø̄r neben $s \bar{a} r$ ist umlautsform,

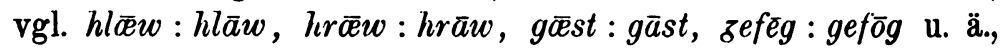
sie erscheint noch wiederholt im Me. nach Stratmann-Bradlay (s. sār subst. und adj.). - Eine neue deutung der inschrift zu geben, bin ich leider nicht im stande: was Boer in dieser

1) Upsala 1900.

2) An English Miscellany, Oxford 1901, p. 362 ff.

3) Marburg 1900. 
beziehung im Arkiv for nordisk filologi 27, 215 ff. 1) vorbringt, mufs ich rundweg ablehnen.

IV. Die alliterierende vorrede $z u$ Wærferðs übersetzung von Gregors Dialogen.

(Vgl. Krebs, Angl. III, 70 f.; Keller, Die litterar. Bestrebungen von Worcester (QF. 84) s. 6 u. 92 f.; Holthausen, Herr. Arch. CV, 367 ff.; Bischofs (!) Wærferth von Worcester Übersetzung der Dialoge Gregors des Grofsen .... herausgeg. von H. Hecht, Leipzig $1900=$ Bibl. der ags. Prosa V.)

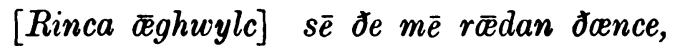
he in me findan mag, gif hine feola lysted gāstlices līfes gōdre bysene, poet he ful eape moeg upp gestīgan

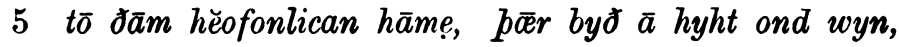
blis on burgum bām be běarn godes sǔelfes ȟ̃ora eagum geseon mōtan. paet maeg se mon begytan, se be his mōdgeđanc aeltowe byp ond ponne purh his ingehygd

10 tō pissa häligra helpe geliefed ond hrora bisene fulgāđ, swā peos bōc sagad.

Me āwrītan hêt Wulfstān bisceop, beow ond pearfa paes be alne prym $\bar{a}[h] \delta f$ ond eac walden [d] is wihta gehwelcre,

15 an éce god ěallra gescěafta.

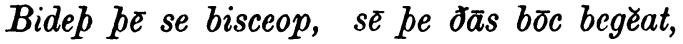

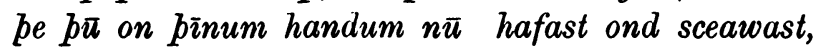
paet pa him tō b̌ossum hālgum helpe bidde, pe heora gemynd hēr on gemearcude š̌endon,

20 ond pat him [grarolīce] god allmihtig forgyue $p \bar{a}$ gyltas, be he ge[o] worhte, ond eac resðe mid him, sē $\circlearrowright e ~ \bar{a} h$ éalles rīces gewerald, ond eac swa his beahgifan, pe him Jās bysene forgěaf, paet is se selesða sinces brytta,

25 EElfryd mid Englum, ěalra cyninga

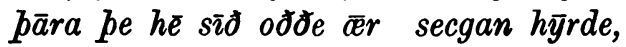

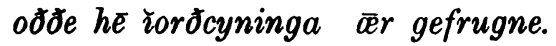

1 bance hs. 3 biesene $h s .5$ parhs. 26 ær fore hs. 27 hiorð $\|$ ær ænigne hs.

1) Vgl. das referat im Jahresber. der germ. Phil. 33. jahrg., XIX, A 1. 


\section{Preis Ealdhelms ( $f$ 709).}

Die folgenden verse in einer hand des 10. jahrh. wurden zuerst von Wanley, Catal. p. 110, dann von Napier, Old English Glosses, Oxford 1900, p. XIV f. veröffentlicht. Sie sind bemerkenswert wegen der zahlreichen eingestreuten lateinischen und griechischen wörter, die natürlich die mittelalterliche (resp. noch moderne) schulaussprache zeigen. Die hs. gehört dem Corp. Chr. Coll. zu Cambridge an und trägt die nr. 326.

bus mē gesette sanctus et iustus, běarn bōca gleaw, bōnus auctor, Ẽaldhelm, apele scěop, etiam füit ipselos on ēঠle Angolsĕaxna,

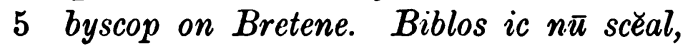
pōnus et pondus plèno cum sensu, ž̌onzes geanode geōmres iamiamque, secgan sōđ, nalles leas, bat him symle waes euthenia oftor on fylste,

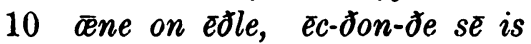
yfel on gesïed. Etiam nusquam ne $s[c] e a l$ lādigan lābor quem tēnet encratea, ac he ěalneg scěal bōethia biddan gěorne

15 purh his mödes gemind micro in cosmo, pat him drihten gyfe dinam[i]s on ̌orðan, fortis factor, poet he ford simle.....

Die griech. wörter hat schon Napier erklärt: v. 4 ipselos $=v \psi \eta \lambda o_{s}$ 'hoch, erhaben', 5 biblos $=\beta i \beta \lambda o s$ 'buch', 6 ponus

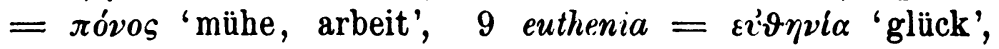
10 cene $=\alpha i \nu \eta$ 'ruhm', 13 encratea $=$ ' $\gamma x \rho \alpha \dot{\tau} \tau \varepsilon \iota \alpha$ 'ausdauer, mäIsigung', 14 boethia $=\beta o \eta \vartheta_{\varepsilon \iota \alpha}$ ' hülfe', 15 micro in cosmo

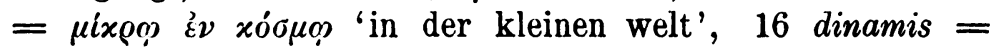

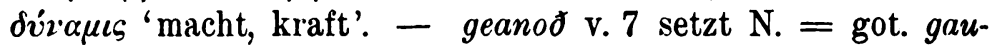
nöbus 'klage, trauern', für $s \bar{E}$ v. 10 würde man eher him erwarten.

\section{Hālgung-bōc.}

Die folgenden 11 verse finden sich in der hs. Cott. Claud. A III, fol. $31 \mathrm{~b}$ aus der ersten hälfte des 11 . jahrh. und wurden

4 æðel hs. || ange̊l hs. 
zuletzt veröffentlicht von Napier, Contributions to Old English Lexicography (in den Philolog. Soc. Transactions 1906) s. 35.

Ic eom hālgungbōc, he̋lde hine dryhten, be me foegere pus froetewum belegde;

$\bar{p} \bar{u}$ ređ tō pance pus hèt mē wyrcean tō loue and to wurde pām pe leoht gesceōp;

5 gemyndi is he mihta gehwylcre pres be he on foldan gefremian moeg and him gebancie peoda waldend, baes pe he on gemynde mädma manega wyle geméarcian metode tō lāce;

10 and he scěal ęce lean ěalle findan baes be he on foldan fremap to ryhte.

Anmerkungen. hālgungbōc ist nach Napier ein 'benedictional', d. h. ein buch, das die kirchlichen segensformeln enthält; es fehlt in allen wörterbüchern, aufser bei Lye. Bemerkenswert ist der nordische name $\bar{p} \bar{u}$ re $ठ$ v. 3 (= aisl.

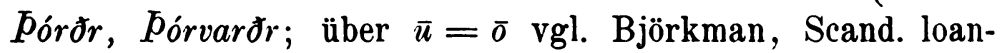
words s. 180).

KIEL.

F. Holthausen. 